Pragmatic and Conversational Features of Arabic-Speaking Adolescents with Autism Spectrum Disorder (ASD): Examining performance and caregivers' perceptions

Almehmadi, Wesam; Tenbrink, Thora; Sanoudaki, Eirini

Journal of Speech, Language and Hearing Research

DOI:

https://doi.org/10.1044/2020_JSLHR-19-00265

Published: 20/07/2020

Peer reviewed version

Cyswllt i'r cyhoeddiad / Link to publication

Dyfyniad o'r fersiwn a gyhoeddwyd / Citation for published version (APA):

Almehmadi, W., Tenbrink, T., \& Sanoudaki, E. (2020). Pragmatic and Conversational Features of Arabic-Speaking Adolescents with Autism Spectrum Disorder (ASD): Examining performance and caregivers' perceptions. Journal of Speech, Language and Hearing Research, 63(7), 23082321. https://doi.org/10.1044/2020_JSLHR-19-00265

\footnotetext{
Hawliau Cyffredinol / General rights

Copyright and moral rights for the publications made accessible in the public portal are retained by the authors and/or other copyright owners and it is a condition of accessing publications that users recognise and abide by the legal requirements associated with these rights.

- Users may download and print one copy of any publication from the public portal for the purpose of private study or research.

- You may not further distribute the material or use it for any profit-making activity or commercial gain

- You may freely distribute the URL identifying the publication in the public portal ?
}

Take down policy

If you believe that this document breaches copyright please contact us providing details, and we will remove access to the work immediately and investigate your claim. 
Pragmatic and Conversational Features of Arabic-Speaking Adolescents with Autism Spectrum Disorder (ASD): Examining performance and caregivers' perceptions

\author{
Wesam Almehmadi ${ }^{1}$, Thora Tenbrink ${ }^{2} \&$ Eirini Sanoudaki $^{3}$ \\ ${ }^{1}$ School of Languages, Literatures \& Linguistics, Bangor University, College Road, Bangor, \\ Gwynedd, LL57 2DG, UK. wesamalmehmadi@hotmail.com
}

${ }^{2}$ School of Languages, Literatures \& Linguistics, Bangor University, College Road, Bangor, Gwynedd, LL57 2DG, UK. t.tenbrink@ bangor.ac.uk

${ }^{3}$ School of Languages, Literatures \& Linguistics, Bangor University, College Road, Bangor, Gwynedd, LL57 2DG, UK. e.sanoudaki@bangor.ac.uk

*Corresponding Author: Wesam Almehmadi

Email: wesamalmehmadi@hotmail.com

Disclosure: The authors declare that there is no conflict of interest.

Funding for this research was provided by the Saudi Ministry of Education, grant no. 143414040-2, awarded to Wesam Almehmadi for her PhD study. 


\begin{abstract}
Purpose: This study investigates the features of pragmatic and conversational skills in the language of Arabic-speaking adolescents with Autism Spectrum Disorder (ASD) by comparing them with typically developing (TD) Arabic-speaking adolescents in Saudi Arabia. It aims to identify the differences in the pragmatic skills of the two groups, and the perception of those skills by caregivers, with respect to four main pragmatic areas: discourse management, communicative function, conversational repair and presupposition abilities.
\end{abstract}

Method: Data for this study were collected from 15 Saudi adolescents with ASD and a control group of 15 TD adolescents, matched for gender and language abilities. All the participants were in the normal IQ range. The caregivers of the adolescents with ASD and TD also participated in this study. Data were collected on the adolescents' performances using the Yale in vivo Pragmatic Protocol (YiPP). In addition, the Pragmatics Profile of Everyday Communication Skills (PPECS) was used to collect data on the caregivers' perceptions of the adolescents' abilities. The combination of tools in this study allows for a unique comparison between actual performance and caregivers' perceptions.

Results: As expected, both the adolescents' performances and the caregivers' perceptions reflected an overall deficit in the pragmatic and conversational skills of adolescents with ASD. However, we also identified an inconsistency between the caregivers' estimation of the participant's pragmatic abilities and the actual abilities demonstrated by the adolescents. In particular, TD adolescents performed significantly better than adolescents with ASD in the pragmatic areas of turn taking, topic maintenance, and topic initiation, but the caregivers did not detect differences between the two groups in these discourse management abilities.

Conclusions: This study has important implications for both ASD interventions and assessment. It provides a comprehensive assessment approach for measuring pragmatic skills, including both direct (participants' performances) and indirect (caregivers' perceptions) 
measures. Future research may benefit from adopting the combined approach used in this study to explore pragmatics in ASD. Differences between caregivers' perceptions and the performances of individuals with ASD should be considered, as well as the influence of various factors on their communication.

Key words: Autism Spectrum Disorder; pragmatics ability; pragmatic assessment; YIPP; PPECS; adolescence; discourse management; communicative functions; conversational repair; presupposition 


\section{Pragmatic and Conversational Features of Arabic-Speaking Adolescents with Autism \\ Spectrum Disorder (ASD): Examining performance and caregivers' perceptions}

\section{Introduction}

Autism Spectrum Disorder (ASD) is a complex developmental disorder characterised by deficits in social communication and interaction, along with restricted interests and repetitive behaviours (American Psychiatric Association, APA, 2013). These issues are interrelated and can negatively affect multiple aspects of one's life, including one's education and social and professional lives (Eaves \& Ho, 2008). ASD also affects many cognitive functions, including the production and understanding of language. Individuals with ASD suffer from noticeable deficits in their language skills, especially in terms of the social use of language for communicative purposes (i.e., pragmatics) (Volden, 2017).

Pragmatic deficit is a hallmark of autism, unlike other language skills that vary from one individual with ASD to another (Tager-Flusberg et al., 2005; Volden, 2017). Volkmar et al. (1997) defined pragmatics as the speaker's ability to use language properly in social contexts and the ability to organize thoughts, using the appropriate social code for the situation to create understanding for the listener. Knowledge of the symbols and grammatical rules of a language is not sufficient to converse successfully or to establish reciprocal social relations. These require the ability to understand the contextual cues and intentions of the participants in a conversation or social interaction, a complex ability that is typically impaired in individuals with ASD (Zufferey, 2015).

Pragmatic deficit among people with ASD becomes clearer in adolescents and adults, as they are more likely to have sufficient knowledge of other language skills, such as 
grammar and vocabulary (Baltaxe, 1977). Adolescence is an exceptional period of considerable growth and development. During this stage of development, individuals often face difficulties coming to terms with various cognitive, physical, and emotional changes. However, this stage is even more challenging for adolescents with ASD (and their families).

This period is also considered a transition period from childhood to adulthood (Seltzer et al., 2003; Barnhill et al., 2000). This transition process often puts a challenge on those with ASD as they will face more social relationships, more academic requirements and increased demand of independent living (Kapp et al., 2011). During adolescence, individuals with ASD become more aware of differences between themselves and typically developing (TD) peers. As one example of these differences, at this stage, TD adolescents tend to use more slang in their conversations whereas many individuals with autism use a more formal, pedantic style of speech, which is, in most cases, not appropriate to the conversational contexts (Whitmire, 2000). This causes them to be excluded from some social activities with peers and negatively affects their ability to make friendships and interact socially with others (Whitmire, 2000).

In addition, language among adolescents with ASD is different from the language of children with ASD, because linguistic abilities in general, even in typical development, continue to change from one stage to another. For instance, some pragmatic abilities, such as understanding figurative language and implicature that require the listeners to go beyond literal meaning and to understand speakers' intended meaning, take more time to master than other abilities that develop in early childhood, such as using speech acts and fundamental conversation abilities (e.g., turn-taking) (Airenti, 2017; Falkum, 2019).

Various difficulties in pragmatic abilities have been widely reported in individuals with ASD and extensively studied (De Villiers et al., 2007; Paul et al., 2009). The most common problems with pragmatics in the population with ASD include difficulties in 
recognising the communicative intention, problems in maintaining conversations, limitation and repetition of topics, sudden switches between topics, echolalia (i.e. repetition of immediate or delayed speech), and pronoun reversal (Paul et al., 2009; Tager-Flusberg et al., 2005; Paul, 2001).

Out of many and varied problems that have been identified in relation to pragmatic skills in ASD, this study focuses on the conversational abilities of adolescents with ASD in four basic areas: discourse management, communicative functions, conversational repair, and presupposition. The focus on these four pragmatic skills derives from the fact that they are the main skills a person needs in order to conduct a successful conversation and interact with others (Simmons et al., 2014). The areas can be specified as follows.

First, one of the main pragmatic difficulties faced by individuals with ASD concerns a deficit in discourse management ability. Discourse management includes the ability to follow the social rules of interaction within a particular conversational context, involving, for example, the ability to take turns, maintain the continuity of the conversation by providing relevant information, and the ability to initiate a new conversational topic (Landa, 2005). Difficulties in different skills required for discourse management are common among individuals with ASD. For example, they tend to have problems recognising communicative intentions and contributing new information to new topics. They may also have difficulties questioning or answering in an appropriate manner, understanding cues to take turns or taking turns at the right times and understanding what is acceptable in a particular culture or society, which results in problems with politeness (Baltaxe, 1977; Tager-Flusberg et al., 2005; Paul et al., 2009; Volden, 2017).

Second, it has been pointed out that individuals with ASD have difficulties expressing communicative functions with words. Basic speech acts, such as questions, requests and 
commands, are acquired by young children in early stages of typical development as part of their knowledge of any language (Airenti, 2017; Kasher, 1991). However, some individuals with autism have problems acquiring this type of pragmatic knowledge. They also have issues dealing with more advanced speech acts, such as congratulating, proclaiming, and promising; and they may become extremely echolalic in such cases (Kasher \& Meilijson, 1996).

Third, individuals with ASD tend to struggle with conversational breakdown and repair (Simmon et al., 2014; Volden, 2017). Conversational repair is a complicated task that demands the successful implementation of a set of cognitive, linguistic, and social skills (Volden, 2004). In discourse, it is important for speakers and listeners to collaborate to ensure the delivery of meaning. Both speakers and listeners share the responsibility to engage in meaningful social exchange. However, if one of the discourse participants fails to keep the exchange meaningful, a communication breakdown occurs and the need for repair and clarification arises. Stirling et al. (2007) suggested that some individuals with autism often encounter problems repairing conversational breakdowns and may require multiple attempts to succeed.

Fourth, individuals with ASD have been reported to experience issues with presupposition ability (Young et al., 2005). Lyons (2013, p. 2356) defines presupposition as "an implicit assumption about the background knowledge relating to an utterance whose truth is taken for granted in discourse". This background knowledge is expected to be known by all participants in a conversation and thus provide information that is appropriate to the communication context (Landa, 2000; Young et al., 2005). Presupposition competence requires the speaker to consider different aspects of the communication context and other conversational participants, in terms of their relationship and background information for a conversational topic (Volden, 2017). The speaker needs to estimate the amount of 
information required, what the listener already knows, and how much remains to be communicated, as well as infer the appropriate type and form of language to be used in the context and topic of the conversation (Landa, 2005; Volden, 2017). Individuals with ASD commonly experience difficulties in their ability to engage in presuppositions (Young et al., 2005). This deficit in presuppositional skills in individuals with ASD can be linked to several factors, including their lack of understanding of verbal and non-verbal cues, and consequently, their inability to produce language in a way that is suited to dynamic context cues (Landa, 2000).

This study addresses these four areas in adolescents with ASD in Saudi Arabia, a population that has not been investigated in this way before. While disorders such as autism are not directly related to a specific culture or language, culture has a significant influence on pragmatic conventions (Leech, 1983). To cooperate successfully with others in their cultural and linguistic environment, children require efficient pragmatic skills that are appropriate for their specific situation (McKibbin \& Hegde, 2011). Pragmatic language and social communication are human behaviours that are closely related to social context and are influenced by cultural variations. The use of different pragmatic behaviours - such as speech acts, politeness, addressing terms, and discourse rules - are considered universal phenomena; yet, each language has its own ways of expressing pragmatic behaviours (Farghal \& Almanna, 2014). For example, people's use of different discourse rules—such as topic choice, turn taking and interrupting during conversation—is typically controlled by cultural rules (Norbury \& Sparks, 2013). To avoid cultural bias, these cultural variations must be considered when investigating pragmatic language, and when designing new tools and when adopting existing tools to assess speech and language (Carter et al., 2012). This motivates studies with a focus on individuals with ASD in countries that have been less explored in this respect. 
Arab countries, specifically Saudi Arabia, are characterised by substantial cultural differences as compared to the English speaking countries in which most previous research on ASD has been conducted. Examples include substantial differences with respect to using some speech acts, gender roles, as well as terms of address and politeness conventions in social hierarchies (Farghal \& Almanna, 2014; Al-Zoubi \& Al-Hassnawi, 2001). As an example of difference in expressing the act of apology, native English speakers (particularly American) tend to use apology using direct speech acts, such as sorry or excuse me, whereas in Arabic, people tend to give more explanations and justifications as basic indirect apology strategies (Ghawi, 1993).

A significant challenge facing individuals with ASD in Saudi Arabia is the lack of awareness among people who share close relationships with them, such as parents and teachers, as well as lack of public awareness of ASD. Lack of knowledge and understanding of the different behaviours, symptoms, and needs associated with ASD may have negative impact on seeking diagnosis, help, and treatment for individuals with ASD. This lack of understanding among some families may also lead to denying their children's disability which hinders the process of their diagnosis and their development (Almasoud, 2013). Some parents of individuals with ASD in Saudi Arabia are more likely to avoid having their children socialise with others because of the lack of public awareness about their situation, which may cause harsh judgment of them (Mashat et al., 2014; Alsehemi et al., 2017). Therefore, most of their interactions and communication with others take place in their schools and with their teachers and school friends.

However, some teachers in Saudi Arabia also lack the ability to recognise the symptoms of autism in their students, which may delay diagnosis and support (Almasoud, 2011). In addition, insufficient training available for teachers and instructors and their lack of experience and awareness of how to identify and address the educational needs of individuals 
with ASD hinder the inclusion of children with ASD in regular schools in Saudi Arabia. Some schools in Saudi Arabia are unable to accommodate these children due to this lack of teachers' knowledge and facilities in public schools, (Almasoud, 2011).

Continued research into difficulties that individuals with ASD experience within the Saudi setting may help to close the gap between current research in the available literature and the practical help that learners with ASD need as well as the advice people working and living with individuals with ASD may require. However, to date, studies have not examined pragmatic difficulties of Arabic-speaking adolescents with ASD at the conversational level, particularly in the Saudi context. Therefore, this study addresses language abilities of Arabic-speaking adolescents in Saudi Arabia with ASD, with a focus on the pragmatic aspects of language, towards establishing a knowledge base that facilitates further research as well as support for individuals with autism in these communities.

Continued research into difficulties that individuals with ASD experience within the Saudi setting may help to close the gap between current research in the available literature and the practical help that learners with ASD need as well as the advice people working with individuals with ASD may require. However, to date, studies have not examined pragmatic difficulties of Arabic-speaking adolescents with ASD at the conversational level, particularly in the Saudi context.

Finally, as it is commonly known, measuring pragmatic language ability is a difficult task, as pragmatics refers to language in context, which is difficult to measure directly (Adams, 2002; Volden et al., 2009). Multiple instruments have been developed to evaluate pragmatic and conversational skills among different populations including direct measures of the actual behaviour of the participants themselves, and indirect measures collected from parents, teachers, clinicians and caregivers of the participants. Direct measures such as 
observations and conversations provide in-depth analyses of participants' language skills and real language performance highlighting contextualised pragmatic skills that are not captured by questionnaires or standardised measures. Whereas, the indirect measures such as parents' questionnaires is useful in providing rich insights into participants' daily behaviour in an authentic environment, home or school without being affected by any variations that may occur in participants' behaviour from day to day (Bishop \& Baird, 2001; Constantino et al., 2003; Volden \& Phillips, 2010). In fact, both tools are useful measures in assessing pragmatic language and identifying language deficit and in order to develop a more comprehensive understanding of these abilities, it is advisable to employ multiple measures and different perspectives when evaluating pragmatic impairment in the ASD population (Condouris et al., 2003). Such a multi-method approach might be the most accurate way to assess and investigate linguistic and pragmatic abilities among individuals with ASD (Luyster et al., 2008).

Despite these various indications in the literature pointing to the benefits of combined approaches in assessing pragmatic and conversational skills, to our knowledge only a small number of studies (Reichow et al., 2008; Volden \& Philips, 2010; King \& Palikara, 2018) have directly combined caregivers' estimations of individuals' pragmatic abilities with those same individuals' actual performance. Our study sets out to address this, in the context of Saudi-Arabian culture. Therefore, this study addresses language abilities of Arabic-speaking adolescents in Saudi Arabia with ASD, with a focus on the pragmatic aspects of language, towards establishing a knowledge base that facilitates further research as well as support for individuals with autism in these communities.

In brief, this study investigates: 
- The conversational skills of Saudi-Arabian adolescents with ASD as compared to those of typically developing (TD) adolescents with respect to discourse management, communicative function, conversational repair, and presupposition abilities. In line with earlier findings, participants with ASD are expected to lack some of the abilities of TD participants.

- The conversational skills of Saudi-Arabian adolescents with ASD as compared to those of TD adolescents according to their caregivers' perceptions. Again, the caregivers' perceptions should highlight that participants with ASD lack of some of the TD participants' abilities.

- A comparison between these two measures. The perceptions of the caregivers are expected to align systematically with the observed strengths and weaknesses of the participants with ASD pragmatic and conversational abilities.

\section{Method}

This study employs mixed methods, between-subjects design to identify and understand the pragmatic and conversational skills inherent in the language of adolescents with autism spectrum disorder (ASD) by comparing them to typically developing (TD) adolescents in Saudi Arabia.

Permissions were obtained from special needs centres in Saudi Arabia to conduct the study with their students. Also, permissions from typically developing adolescents' families were obtained in order to gather data from their sons and daughters. Upon receiving their permission, ethical approval was provided by Bangor University's research ethics committee (No. CAH 37). Informed consent was obtained by caregivers/ parents prior to testing.

\section{Participants}


Thirty native Arabic speakers between the ages of 10 - 17 years participated in this study and divided into two groups: ASD group and typically developing (TD) group. The participants' details are shown in Table 1. Fifteen of the participants were adolescents with ASD, and the other 15 were typically developing (TD) adolescents. There were five female and ten male participants in each group. Although a higher number of participants would be beneficial for quantitative analysis, recruiting participants with ASD poses major challenges. Consequently, the number of participants in most published studies on autism ranges from 10 to 30 (Whitman, 2004).

Fifteen caregivers from each participating adolescent group (ASD and TD) were recruited as well to complete a questionnaire about the adolescents' pragmatic behaviours and difficulties. In the TD group, the parents completed the questionnaire; whereas in the ASD group, the teachers completed the questionnaire. The reason for the difference between the caregivers in each group was that permission to access public schools to collect data from the teachers of the TD adolescent participants was not granted and some of the parents of the adolescents with ASD were not willing to participate.

However, the different roles the two informant groups (parent and teachers) play in the participants' life may not have limited their ability to provide reliable ratings of the participants' pragmatic abilities. Both the teachers and the parents communicate with the participants on a (almost) daily basis and observe them in different contexts and in interaction with others (e.g., friends or family members). Moreover, as the adolescents with ASD studied in centres dedicated to people with special needs; thus, their teachers' role is not limited to teaching them academic skills but also includes improving their social and communication skills, making them credible sources of information about the participants' different abilities.

The adolescents with ASD were recruited from special needs centres in the cities of Mecca and Jeddah, in the western region of Saudi Arabia. At the time of the study, the 
participants were not institutionalised in private-care facilities but were living with their families and attending special programmes in private-care centres for people with autism and special needs. The participants in the comparison group (TD) were selected through community resources and local social groups.

The inclusion criteria for the ASD participant group were: age (10 - 17 years old); ASD diagnosis; no additional diagnoses or disabilities, such as sight or hearing loss; and IQ within the normal range $(85-104)$. For the TD group, the inclusion criteria were age $(10-$ 17 years old), typical development, no history of a developmental disorder, and IQ within the normal range. For each group, 15 caregivers were chosen to complete a questionnaire about the adolescents' pragmatic behaviours and difficulties.

All participants in the ASD group already had well-established diagnoses of autism spectrum disorder by specialist clinicians. The two groups were equivalent in terms of gender as well as native language and culture (Arabic). For the purpose of this study, the participants were also matched in terms of verbal ability by using an adaptation of the Test for the Reception of Grammar (TROG) (Bishop, 2003) which measures receptive grammar and of the British Picture Vocabulary Scales (BPVS) (Dunn \& Dunn, 2009) which measures receptive vocabulary. In addition, participants had nonverbal IQs in the average range (85 104) as measured by Kaufman Brief Intelligence Test (KBIT) (Kaufman and Kaufman, 2004). Independent sample $t$-tests showed that the two groups did not differ statistically on receptive grammar, TROG, $\mathrm{t}(29=-0.940, p=.355$, and receptive vocabulary, BPVS, $\mathrm{t}(29)$ $=0.069, p=.946$. However, the two groups could not be perfectly matched with respect to nonverbal IQ, $\mathrm{t}(29)=-4.977, p=.001$, where the TD group scored slightly but significantly higher (100 as opposed to 94).

[Table 1 about here] 


\section{Materials}

\section{Yale in vivo Pragmatic Protocol (YiPP)}

A translated and adapted version of YiPP (Simmons et al., 2014) was used in this study. This instrument consists of a semi-structured conversation that includes a set of probes testing the participants' conversational behaviours in the four conversational areas targeted in this study: discourse management, communicative function, conversational repair and presupposition. There are 19 probes for these four areas, each of which is designed to stimulate a certain pragmatic behaviour. The protocol is designed to appear as natural as possible to the participants, in spite of following a script targeting a 30-minute conversation.

The participants' responses to each pragmatic probe were recorded as error scores and as cue scores. The error scores indicate whether or not the participant's answers to the probes were appropriate. A zero score indicates that the answer provided was compatible with the desired response; a score of one suggests that the answer was somewhat inappropriate while a score of two means that the answer was not suitable or cannot be considered a response. The cue scores determine to what extent the examiner provided cues to the participants when their answer was wrong, or when they did not provide any answers. These scores range from zero to six. The lowest score on this scale, which is zero, means that the participant did not give an answer in spite of all the hints that were provided to him or her. The highest score, six, indicates that the participant responded in an appropriate manner without any help or hints. Obtaining a high score in the error scores is a sign of a weak performance by the participant, while the highest score in the cue score is evidence of an appropriate performance (see table 2). The participants' conversations were audio recorded and later scored and analysed by the researcher (first author). However, some notes were taken during the conversations with the participants, especially when presenting the non-verbal cues, as permission to use video recording was not granted. The data were then checked independently by a second researcher, 
who was naive as to which participants had an ASD diagnosis. The two raters agreed on most items, and where they didn't they played the recordings again to ensure appropriate scoring in every case.

[Table 2 about here]

\section{Pragmatics Profile of Everyday Communication Skills in Adults, (PPECS)}

This study also used a modified and translated version of the PPECS developed by Dewart and Summers (1996). This profile has been used in a number of publications to investigate communication and pragmatic abilities in a wide range of individuals including children with Down's syndrome (Johnston \& Stansfield, 1997), children with autism and epilepsy (Parkinson, 2006), children with William Syndrome (Stojanovik \& James, 2006) and deaf children (Mouvet et al., 2013). It was used as a mean of involving the caregivers of the children who are in constant contact with them, so as to evaluate and identify children's communication and language abilities in everyday life outside the clinical environment. The main format of the original profile was converted from a semi-structured interview to a questionnaire for the purposes of the current study. It includes two pragmatic behaviours which are: communicative functions, and interaction and conversation. The modified questionnaire consists of 22 questions which examine different pragmatic behaviours, and it includes different types of communicative behaviours under each question as multiple-choice options which are suited to a range of developmental levels. These options are related to individuals' behaviours and reactions in certain situations. They are at different degrees of functioning, and range from the typical behaviour at the top to the least typical at the bottom of the multiple choices. Higher scores are a sign of atypical behaviour and greater difficulties and the maximum is $100 \%$. 


\section{Categories used in the tools}

The YIPP and PPECS tools focus on almost the same pragmatic behaviours using different classifications in some behaviours. Specific pragmatic behaviours in the two tools are chosen to measure the same pragmatic areas from two different points of view, the participants' performances and their caregivers' perceptions. In YIPP, there are four main pragmatic domains: discourse management, communicative function, conversational repair and presupposition. In PPECS, there are only two main pragmatic domains: communicative functions, and interaction and conversation. Here, the interaction and conversation domain includes some pragmatic abilities as those in YIPP, including conversational repair, presupposition and some discourse management abilities such as initiation, maintaining an interaction, joining a conversation (turn-taking) and terminating a conversation.

\section{Translation of data collection tools}

The tools used in this research were adapted and translated from English into Arabic to ensure reliability and clarity and to guarantee that they achieve their intended purpose. The first author used back translation, a common and recommended method of translation, to increase the accuracy of the translation. The first author translated the instruments used from English into Arabic, as she is a native Arabic speaker, with sufficient fluency in English and familiarity with the research topic. The translated tools in Arabic were then given to three Arabic-English bilingual speakers to independently translate the tools back into English. A comparison between the two versions was subsequently made to clarify issues and remove ambiguities in the Arabic version and an Arabic language version was reached for each tool. This Arabic version was then sent to a well-known Arabic teacher in this field, who reviewed the tools, edited mistakes, checked spelling and grammar, and created a final version of the instruments. Finally, a pilot study was conducted to test the validity of these translated tools. 


\section{Procedures}

The study was carried out in a series of sessions. Language tests (BPVS and TROG) were administered first, followed by the IQ test (KBIT) on the same day. There was a break after each test. In a separate session on a different day, a warm-up conversation took place for about 10 minutes to give the participants a sense of comfort and familiarity toward the researcher. Then, the researcher gradually moved on to the YIPP conversation to make it seem natural for participants. The YiPP took approximately 20 - 30 minutes. All conversations were audio recorded. While the researcher and the participants engaged in the previous tasks, parents and caregivers were given the PPECS questionnaire to complete, which took about 10 - 15 minutes.

To ensure participants' concentration, testing took place in a quiet room with comfortable chairs and a table. Anything that might cause distractions for the participants was removed.

\section{Statistical analysis}

All assessment data were analysed using SPSS version 23.0 (IBM, 2015). To investigate group differences in the two main tools (YIPP and PPECS), analysis of covariance (ANCOVA) and multivariate analysis of covariance (MANCOVA) were used. Follow-up univariate analyses of covariance for each variable were also conducted to identify items where the groups differed. Since participants differed on their IQ scores, IQ was added to the models as a covariate to ensure that any difference between the groups in the final results was not due to differences in this background factor. Bonferroni correction was used to correct for multiple testing.

\section{Results}


Since ANCOVA analysis showed no significant gender differences in either one of the groups (ASD and TD) across all pragmatic domains in PPECS and in YIPP, subsequently results for female and male participants were collapsed.

\section{YIPP statistical analysis}

\section{- Four pragmatic YIPP domain results}

In YIPP, the participants' responses to each pragmatic probe were recorded as error scores and as cue scores. As expected, the performance of the TD participant group was better than that of the group with ASD in both error and cue scores, as evidenced by their lower means in error scores and higher means in cue scores (see Figures 1 and 2).

After adjustment for nonverbal IQ, there was a significant difference between the two groups in their error scores in the four pragmatic domains in YIPP, as follows: discourse management, $\left[F(1.27)=110.64, p<.001, \eta p^{2}=.80\right]$; communicative function, $[F(1.27)=$ $\left.6.121, p=.020, \eta p^{2}=.18\right]$; conversational repair, $\left[F(1.27)=107.27, p<.001, \eta p^{2}=.79\right]$; and presupposition, $\left[F(1.27)=41.99, p<.001, \eta p^{2}=.60\right]$ such that the TD group performed better than the ASD group.

[Figure 1 about here]

In addition, in the cue scores, there was a significant difference between the two groups in the four pragmatic YIPP domains, as follows: discourse management, $[F(1.27)=$ 88.07, $\left.p<.001, \eta p^{2}=.76\right]$; communicative function, $\left[F(1.27)=13.02, p=.001, \eta p^{2}=.32\right]$; conversational repair, $\left[F(1.27)=54.55, p<.001, \eta p^{2}=.66\right]$; and presupposition, $[F(1.27)=$ 65.78, $\left.p<.001, \eta p^{2}=.70\right]$, such that the TD group performed better than the ASD group. Means and standard deviations of error and cue scores of the four pragmatic domains are shown in Table 3. 
[Figure 2 about here]

[Table 3 about here]

\section{- Yale in Vivo Pragmatic Protocol (YIPP) probe analysis}

In order to provide a more detailed picture of the participants' pragmatic abilities and to determine the specific probes that differed between each group, a multivariate analysis of covariance (MANCOVA) was run to examine the differences between the mean scores of two groups (adolescents with TD and ASD) for each of the 19 probes of YIPP, which is designed to stimulate a certain pragmatic behaviour. IQ scores were added as covariates in the analyses.

With IQ scores as the covariates, the multivariate effects were significant for both error scores $\left[F(19.9)=26.21, p<.001, \eta p^{2}=.982\right]$ and cue scores $[F(19.9)=39.23, p<$ $\left..001, \eta p^{2}=.988\right]$. This result indicated that there was an overall significant difference between participants with TD and ASD where the TD group performed better than the group with ASD. Follow-up univariate analyses of covariance for each variable were also conducted to identify probes where the groups differed. The data for the error and cue scores from these analyses are summarized in table 4 (significant differences are marked by an asterisk *). In addition, table 5 presents the adjusted means for both groups' error and cue scores for each probe.

[Table 4 about here]

[Table 5 about here]

\section{Pragmatic Profile of Everyday Communication Skills (PPECS) statistical analysis}

- Two pragmatic PPECS domains results 
ANCOVA was carried out to compare participants with ASD and typically developing participants in their conversational skills as perceived by their caregivers and measured by PPECS after controlling for nonverbal IQ.

The caregivers' questionnaire consisted of two pragmatic domains, namely communicative function, and interaction and conversation. After adjustment for nonverbal IQ, there was a significant difference between the two groups in their pragmatic behaviours in both pragmatic domains as follows: communicative function, $[F(1.27)=50.12, p<.001$, $\left.\eta p^{2}=.650\right]$; interaction and conversation, $\left[F(1.27)=5.30, p=.029, \eta p^{2}=.164\right]$ where TD adolescents were better than adolescents with ASD as rated by their caregivers (see table 6 and figure 3).

[Table 6 about here]

[Figure 3 about here]

\section{- Pragmatic Profile of Everyday Communication Skills (PPECS) item analysis}

In order to provide a closer examination of the differences between the groups and to determine the specific items where the groups differed, a MANCOVA was run to examine differences between the responses of caregivers of each group to 22 questionnaire items which examine different pragmatic behaviours. With IQ scores as covariates, the TD group generally scored significantly better on the questionnaire than the group with ASD as rated by their caregivers $\left[F(22.6)=5.02, p=.026, \eta p^{2}=.949\right]$. Follow-up univariate analyses of each variable's covariance were also conducted to identify the items where the groups differed. The data for the item scores from this analysis are summarized in table 7 (significant differences are marked by an asterisk *).

[Table 7 about here] 
Specific pragmatic behaviours in the two tools (YiPP and PPECS) were chosen to measure the same pragmatic skills from two different points of view, the participants' performances and their caregivers' perceptions. Analysis results of the data from the two tools show some similarities and differences in the pragmatic language characteristics of adolescents with ASD compared to TD adolescents based on the two sources of information. The data about the pragmatic behaviours with significant differences between the two groups, from the caregivers' perspectives, the adolescents' performances, or both, is summarised in Table 8 (significant differences are marked by an asterisk *).

[Table 8 about here]

\section{Discussion}

This study investigated the conversational abilities of Saudi Arabian adolescents with and without ASD in actual performance and according to caregivers' perceptions, using a variety of measures. As expected, TD participants performed significantly better than the ASD group both in performance and in their caregivers' perception in the main pragmatic domains. However, each general pragmatic domain as measured by the YIPP and PPECS tools is associated with a number of specific pragmatic behaviours. Closer inspection and analysis of the probes and items within each domain showed significant differences in some abilities but not in others.

Several important insights emerged from the different assessment methods used in this study. Analyses of the data from the two tools show some similarities and differences in the pragmatic language characteristics of adolescents with ASD compared to TD adolescents based on the two sources of information. However, the most important finding is the inconsistency between the caregivers' estimation of the participants' pragmatic abilities and 
the abilities the adolescents demonstrated in their actual performances. For instance, a number of key difficulties that were identified in the performances of adolescents with ASD, including deficits in topic initiation, maintaining conversations, and turn-taking, were not identified by the caregivers, who did not detect differences between the two groups in these behaviours.

During conversations with participants, most adolescents with ASD exhibited difficulties in offering a topic to initiate the conversation when given the chance. They also struggled to maintain the topic of conversation, had difficulties in adding new and relevant information to expand on the conversation topic and keeping the flow. Participants with ASD also demonstrated issues in turn-taking during conversations: some were less responsive to cues that signalled taking a turn, others showed delays in taking turns causing hesitation and pauses, and some tended to monopolise the conversation causing many interruptions during the conversations. In contrast, caregivers thought participants with ASD were able to start conversations with others, maintain the conversational flow, and join in conversations and take turns appropriately.

The conversational difficulties seen in our participants' performance are in line with those reported by a large body of earlier studies on conversational problems in autism, such as Paul and Landa (2008) and Simmons et al. (2014). These studies reported that individuals with ASD faced difficulties engaging in fundamental aspects of conversations, such as initiating and sustaining conversations and taking turns in socially conventional ways. BaronCohen (1988) explained that difficulties in turn-taking in autism may be manifest in different forms, such as inappropriate interruption, the inability to signal turn-taking and holding the speaker or respondent role for too long.

Familiar routines, repetitive actions, and caregivers' support may help individuals with ASD achieve such behaviours and give their caregivers the impression that they have a 
good grasp of these conversational skills. These factors and their influences on the communicative performances of the adolescents with ASD could mask their actual capabilities and affect how their teachers perceive and assess their abilities. However, providing different levels of support to facilitate the communication process and accommodate the different needs of the individuals with ASD may lead their caregivers to overestimate their actual pragmatic skills (Tomasello \& Mervis, 1994; Luyster et al., 2008).

Contrary to the results of this study, Volden and Phillips (2010) in their study on pragmatic difficulties in individuals with ASD found that the assessment of caregivers was more accurate and effective in detecting pragmatic impairment in participants with ASD than measuring actual performance. They combined two types of measures, the Children's Communication Checklist-2 (CCC-2; Bishop, 1998), which allowed the caregivers to report their assessments, and the Test of Pragmatic Language (TOPL, Phelps-Terasaki \& PhelpsGunn, 2007), which measured the participants' actual performance.

The discrepancy between the participants' actual pragmatic behaviour and their caregivers' perceptions found in this study is an important finding. It draws attention to the importance of combining direct and indirect assessment tools to overcome deficiencies that can arise from the use of direct or indirect unaccompanied measures. It also enabled the study to cover a wide range of pragmatic abilities and highlight different aspects of the strengths and weaknesses in the language of the individuals with ASD.

In the current study, the pragmatic abilities of the adolescents with ASD were assessed by their teachers at centres dedicated to people with special needs. Their teachers' role is not limited to teaching them academic skills but also includes improving their social and communication skills. Therefore, their teachers have a fair knowledge of the adolescents' abilities and have adopted certain ways to communicate with them for example, by 
controlling turns in conversations, stimulating communication, and providing more questions and motivation (Basil, 1992; Chiang \& Carter 2008); this may have led the teachers to be more accepting of their students' difficulties and may have caused them to overestimate their students' real abilities. The teachers also communicate with ASD students with a wide range of abilities and difficulties; this may also have affected their ratings as the teachers may have compared a participant with others in the class who have less abilities. As such, the school's familiar environment, with its daily routine and repetitive actions, and the support of their teachers, who may provide different levels of support and cues, may be beneficial in helping the adolescents with ASD to display some communicative skills. However, changing the communicative context or the communication style and introducing a new and unfamiliar situation may bring their difficulties to surface.

The possible overestimation or inaccuracy in the caregivers' assessments found in this study might be related to the study context and the caregivers of adolescents with ASD in Saudi Arabia. As reported in many previous studies, parents and teachers in Saudi Arabia are more likely to lack awareness of autism, its symptoms, causes, and the different needs of individuals with ASD (Almasoud, 2011; Alqahtani, 2012; Alamri \& Tyler-Wood, 2016). In contrast, caregivers in Western cultures were reported as having greater awareness about autism, and the partnership approach between parents and professionals was encouraged and followed in most Western contexts (Ravindran \& Myers, 2012). Different cultural conceptualization about autism and knowledge of parents or care providers might have an impact on the way they perceive and understand different symptoms and behaviours related to autism and in seeking assistance, treatments, and intervention (Alqahtani, 2012; Ravindran \& Myers, 2012). 
The findings from this study support the conclusion that a combined approach, using direct measures that focus on the participants themselves accompanied by indirect assessment obtained through a caregiver questionnaire, is a useful way to measure pragmatic skills and gain insight into the pragmatic abilities of adolescents with ASD (see also Adams, 2002; Condouris et al., 2003). The combined approach used in this study provides empirical support of the benefits and utility of this approach in assessing pragmatic skills in ASD. It is beneficial in avoiding and compensating for the limitations of either one of the assessments, since the caregivers' ratings alone or the participants' behaviours alone may not represent the entire picture of the pragmatic function of the participants.

Using indirect measures in this study (the caregivers' questionnaire, PPECS) was useful in providing insight into the participants' pragmatic behaviours in their daily interactions in authentic environments (home or school); however, these ratings may have been influenced by the subjectivity of the caregivers, who may at times have overestimated the participants' abilities. The caregivers are more familiar and more experienced in dealing with adolescents with ASD, and this may have prevented them from noticing their actual function levels. The use of direct measures of the participants' pragmatic performance, on the other hand, including semi-structured conversations in the YiPP, was beneficial in highlighting the actual, contextualised, pragmatic skills of the participants. However, the performance of the participants was measured in particular situations, and their behaviours varied and fluctuated from day to day and in contact with unfamiliar adults. Using a combined approach in this study, pragmatic skills were measured in terms of the participants' perceived ability in their daily communication during conversations in everyday events as well as in terms of their actual use in a novel context outside their usual routine, avoiding the possible subjectivity of the raters, and assessed without the support of a familiar conversational partner. 
Finally, it is worth mentioning that the discrepancy between the observed and reported abilities of the adolescents with ASD in this study may not only be the result of their caregivers' support or overestimation of their abilities. In fact, it is possible that caregivers' assessments are based on noticing and describing the adolescents pragmatic behaviors across different contexts and in contact with familiar and unfamiliar people in contrast to the direct assessment of their behavior with was done through conversations with an unfamiliar adult in particular situation. Thus, they might describe an actual change and development in adolescents' conversational abilities. Therefore, it would be beneficial in future research to compare data from caregiver's assessment to data collected from participants' conversations when interacting with familiar conversational partners (a family member or a friend) and in different conversational context to investigate the effect of these factors and their influence on the pragmatic functioning of individuals with ASD.

\section{Implications}

Our findings have a range of relevant implications for the field of pragmatics and autism. These results may be useful in both the area of pragmatic assessment as well as in the design of intervention programmes for adolescents with ASD, aimed at developing their conversational skills and social communication abilities.

Apart from the clear benefits of combining assessment tools demonstrated by this study, the identified inconsistency between the caregivers' estimation of the participant's pragmatic abilities and their actual abilities alerts to a need to enhance caregiver sensitivity and support classifying and understanding the particular difficulties experienced by individuals with ASD. Caregivers are an essential source of information in assessing pragmatic abilities in autism and observing the strengths and weaknesses of individuals with ASD, because they have more opportunities to interact with them in a variety of situations 
than anybody else. Therefore, appropriate training, education and support must be provided to caregivers of individuals with ASD to increase caregiver knowledge and awareness of their children's situation, which could have a positive effect on progress in developing language, communication and social skills, and thus ensure a better life for individuals with autism.

The research findings may also contribute to the design of educational interventions and programmes to promote the development of the abilities of individuals with ASD. The pragmatic difficulties found in the participants' conversations (e.g., difficulties in conversational repair, turn-taking, topic initiation, and presupposition) can be used as the main elements and targets for intervention programmes directed at enhancing the conversational abilities of adolescents with autism.

\section{Future research and limitations}

In this study, we chose to focus on adolescents with ASD whose IQs within the normal range. However, individuals in the autism spectrum differ widely with respect to language and cognitive abilities across different stages of development. Therefore, our results may not be generalisable to all individuals in the autism spectrum; further studies are required to account for the range of variation in ASD, specifically with respect to the possible divergences between caregiver perception and actual performance. It may also be noted that (in line with previous studies) our sample was relatively small; future research (ideally with larger samples) would therefore be useful to gain further insights concerning the validity of the findings.

\section{Conclusion}

This study identified the features of pragmatic and conversational difficulties that are present in the language of adolescents with ASD by comparing them with TD adolescents in Saudi Arabia. Two important findings related to the participants' pragmatic and 
conversational abilities emerged from the results of this study. First, multiple pragmatic issues and a spectrum of conversational difficulties were found in the conversations of adolescents with ASD compared to their typically developing peers. A further major finding concerned the disagreement between participants' actual performance and the rating of their caregivers with respect to some pragmatic skills. The combination of these instruments provides a comprehensive profile of the conversational abilities of individuals with ASD by investigating different conversational difficulties they experience and by comparing the behaviours of individuals with ASD and those of TD individuals from two points of view: actual performance and caretakers' perception by incorporating both methods of assessment.

\section{Acknowledgements}

This article was completed as part of the first author's doctoral dissertation. We thank everyone who contributed to this work, including Bangor University for hosting the $\mathrm{PhD}$ research. We gratefully acknowledge all the participants and their caregivers who took part in the study. We would also like to thank the Autism centres that participated in this research for their invaluable help. Finally, we thank the Saudi Ministry of Education for funding this research.

\section{References}

Adams, C. (2002). 'Practitioner Review: The Assessment of Language Pragmatics', Journal of Child Psychology and Psychiatry, 36, 289-306.

Airenti, G. (2017). Pragmatic development. In: Cummings L, (eds). Research in clinical pragmatics. Cham: Springer International Publishing, 3-28.

Alamri, A. A. \& Tyler-Wood, T. (2016). Teachers' attitudes toward children with autism: a comparative study of the United States and Saudi Arabia. Journal of The International Association Of Special Education, 14-25.

Almasoud, H. (2011). What does prospective teachers need to know about autism?

Evaluation of an autism course in a Saudi university. Master dissertation. Birmingham: The University of Birmingham. 
Almasoud, H. (2013). Educating students with autism spectrum conditions (ASCs) in Saudi Arabia: policy, practice and provision for inclusive and special education. King Saud University. [Online]. Available at:

http://faculty.ksu.edu.sa/sites/default/files/f_policy_practice_and_provision_for_sen_ 3.output.pdf Accessed 12 December 2019.

Alqahtani, M.M. (2012). Understanding autism in Saudi Arabia: a qualitative analysis of the community and cultural context. Journal of Pediatric Neurology, 10 (01), 015-022.

Alsehemi, M.A., Abousaadah, M.M., Sairafi, R.A. \& Jan, M.M. (2017). Public awareness of autism spectrum disorder. Neurosciences, 22 (3), p.213.

Al-Zoubi, M.Q. \& Al-Hassnawi, A.R. (2001). Constructing a model for shift analysis in translation. Translation Journal, 5 (4), 1-37.

American Psychiatric Association, (2013). Diagnostic and statistical manual of mental disorders (DSM-5®). American Psychiatric Pub.

Baltaxe, C. (1977). Pragmatic deficits in the language of autistic adolescents. Journal of Pediatric Psychology, 2, 176-180.

Barnhill, G. P., Hagiwara, T., Myles, B. S., Simpson, R. L., Brick, M. L., \& Griswold, D. E. (2000). Parent, teacher, and self-report of problem and adaptive behaviors in children and adolescents with Asperger syndrome. Diagnostique, 25(2), 147-167.

Baron-Cohen, S. (1988). Social and pragmatic deficits in autism: cognitive or affective? Journal of Autism and Developmental Disorders, 18, 379-402.

Basil, C. (1992). Social interaction and learned helplessness in severely disabled children. Augmentative and Alternative Communication, 8, 188-199.

Bishop, D. V. (1998). Development of the Children's Communication Checklist (CCC): A method for assessing qualitative aspects of communicative impairment in children. The Journal of Child Psychology and Psychiatry and Allied Disciplines, 39(6), 879891.

Bishop, D. V. M., \& Baird, G. (2001). Parent and teacher report of pragmatic aspects of communication: use of the Children's Communication Checklist in a clinical setting. Developmental Medicine and Child Neurology, 43, 809-818.

Bishop, D. V. M (2003). The Test for Reception of Grammar: TROG 2. London: Psychological Corporation.

Carter, J.A., Murira, G., Gona, J., Tumaini, J., Lees, J., Neville, B.G. and Newton, C.R., (2012). Speech and language disorders in Kenyan children: Adapting tools for regions with few assessment resources. Journal of Psychology in Africa, 22(2), 155-169. 
Chiang, H.M. \& Carter, M. (2008). Spontaneity of communication in individuals with autism. Journal of Autism and Developmental Disorders, 38 (4), 693-705.

Condouris, K., Meyer, E., \& Tager-Flusberg, H. (2003). The relationship between standardized measures of language and measures of spontaneous speech in children with autism. American journal of speech-language pathology, 12(3), 349-358.

Constantino, J. N., Davis, S. A., Todd, R. D., Schindler, M. K., Gross, M. M., Brophy, S. L., Reich, W. (2003). Validation of a brief quantitative measure of autistic traits: Comparison of the social responsiveness scale with the autism diagnostic interviewrevised. Journal of Autism and Developmental Disorders, 33(4), 427-33.

Dewart, H., \& Summers, S. (1996). The pragmatics profile of everyday communication skills in adults. Windsor: NFER Nelson.

De Villiers, J., Stainton, R. J., \& Szatmari, P. (2007). Pragmatic abilities in autism spectrum disorder: A case study in philosophy and the empirical. Midwest Studies in Philosophy, 31(1), 292-317.

Dunn, L., \& Dunn, D. (2009). The British Picture Vocabulary Scale manual (3rd ed.). London, England: GL Assessment.

Eaves, L.C. \& Ho, H.H. (2008). Young adult outcome of autism spectrum disorders. Journal of Autism and Developmental Disorders, 38 (4), 739-747.

Falkum, I.L. (2019). Pragmatic development. Learning to use language to communicate. J.S. Horst \& J. von Koss Torkildsen (red.), International Handbook of Language Acquisition. London: Routledge, 234-260.

Farghal, M., \& Almanna, A. (2014). Some Pragmatic Aspects of Arabic/English Translation of Literary Texts. Jordan Journal of Modern Languages and Literature, 6 (2), 93-114.

Ghawi, M. (1993). Pragmatic transfer in Arabic learners of English. The Arizona Working Papers in Second Language Acquisition and Teaching, 1, 39-52.

IBM Corp. Released (2015). IBM SPSS Statistics for Windows, Version 23.0. Armonk, NY: IBM Corp.

Johnston, F., \& Stansfield, J. (1997). Expressive pragmatic skills in pre-school children with and without Down's syndrome: parental perceptions. Journal of Intellectual Disability Research, 41(1), 19-29.

Kapp, S. K., Gantman, A., \& Laugeson, E. A. (2011). Transition to adulthood for highfunctioning individuals with autism spectrum disorders. In A comprehensive book on autism spectrum disorders. InTech, 2011.

Kasher, A., (1991). On the pragmatic modules: a lecture. Journal of Pragmatics, 16:381-397. 
Kasher, A., \& Meilijson, S. (1996). Autism and pragmatics of language. Incontri Cita Aperta, $4,37-54$.

Kaufman, A. S., \& Kaufman, N. L. (2004). Kaufman Brief Intelligence Test (2nd ed.). Bloomington, MN: Pearson.

King, D., \& Palikara, O. (2018). Assessing language skills in adolescents with autism spectrum disorder. Child Language Teaching and Therapy, 34(2), 101-113.

Landa, R. (2000). Social language use in Asperger syndrome and high-functioning autism. In A. Klin, F. R. Volkmar, \& S. S. Sparrow (Eds.), Asperger syndrome, New York, NY: Guilford Press, 125-155.

Landa, R.J. (2005). Assessment of social communication skills in preschoolers. Mental Retardation and Developmental Disabilities Research Reviews, 11 (3), 247-252.

Leech, G. (1983). Principles of pragmatics. London: Longman.Loukusa, S., Leinonen, E., Jussila, K., Mattila, M.L., Ryder, N., Ebeling, H. and Moilanen, I., (2007). Answering contextually demanding questions: Pragmatic errors produced by children with Asperger syndrome or high-functioning autism. Journal of Communication Disorders, 40 (5), 357-381.

Luyster, R. J., Kadlec, M. B., Carter, A., \& Tager-Flusberg, H. (2008). Language assessment and development in toddlers with autism spectrum disorders. Journal of autism and developmental disorders, 38 (8), 1426-1438.

Lyons, M. (2013). Presupposition. In F. R. Volkmar (Ed.), Encyclopedia of autism spectrum disorders (p. 2356). New York, NY: Springer.

Mashat, A., Wald, M. \& Parsons, S. (2014). Improving social and communication skills of adult Arabs with ASD through the use of social media technologies. In: Miesenberger K., Fels D., Archambault D., Peňáz P., Zagler W. (eds) Computers Helping Peop with Special Needs. ICCHP 2014. Lecture Notes in Computer Science, vol 8547. Springer, Cham, 478-485.

Mckibbin, R. C. and Hegde, M. N., (2011). An advanced review of speech-language pathology: Preparation for NESPA and comprehensive examination. 3rd ed. Austin: Pro.ed.

Mouvet, K., Matthijs, L., Loots, G., Taverniers, M., \& Van Herreweghe, M. (2013). The language development of a deaf child with a cochlear implant. Language Sciences, 35, 59-79.

Norbury, C.F. and Sparks, A., (2013). Difference or disorder? Cultural issues in understanding neurodevelopmental disorders. Developmental Psychology, 49(1), 45. 
Parkinson, G. (2006). Pragmatic difficulties in children with autism associated with childhood epilepsy. Pediatric Rehabilitation, 9, 229-246.

Paul, R. (2001). Language disorders from infancy through adolescence: assessment \& intervention. St. Louis, Mosby.Paul, R., \& Landa, R. (2008). Communication in Asperger syndrome. In A. Klin, S. Sparrow, \& F. Volkmar (Eds.), Asperger syndrome (2nd ed.). New York, NY: Guilford Press.

Paul, R., \& Landa, R. (2008). Communication in Asperger syndrome. In: Klin, A., Sparrow, S. \& Volkmar, F. (eds). Asperger syndrome. 2nd ed. New York, NY: Guilford Press.

Paul, R., Orlovski, S., Marcinko, H. C., \& Volkmar, F. R. (2009). Conversational behaviors in youth with high-functioning ASD and Asperger syndrome. Journal of Autism and Developmental Disorders, 39, 115-125.

Phelps-Terasaki, D., \& Phelsp-Gunn, T. (2007). Test of Pragmatic Language (TOPL-2). ProEd.

Ravindran, N. \& Myers, B.J. (2012). Cultural influences on perceptions of health, illness, and disability: a review and focus on autism. Journal of Child and Family Studies, 21 (2), 311-319.

Reichow, B., Salamack, S., Paul, R., Volkmar, F. R., \& Klin, A. (2008). Pragmatic assessment in autism spectrum disorders: A comparison of a standard measure with parent report. Communication Disorders Quarterly, 29, 169-176.

Seltzer, M. M., Krauss, M. W., Shattuck, P. T., Orsmond, G., Swe, A., \& Lord, C. (2003). The symptoms of autism spectrum disorders in adolescence and adulthood. Journal of autism and developmental disorders, 33(6), 565-581.

Simmons, E. S., Paul, R., \& Volkmar, F. (2014). Assessing pragmatic language in autism spectrum disorder: Yale in vivo Pragmatic Protocol. Journal of Speech, Language \& Hearing Research, 57(6), 2162-2173.

Stirling, L., Barrington, G., \& Douglas, S. (2007). Two times three little pigs: Dysfluency, cognitive complexity and autism. In I. Mushin \& M. Laughren (Eds.), Selected papers from 2006 Annual Meeting of the Australian Linguistics Society.

Stojanovik, V., \& James, D. (2006). Short-term longitudinal study of a child with Williams syndrome. International Journal of Communication Disorders, 41, 213-223.

Tager-Flusberg, H., Paul, R., \& Lord, C. (2005). Language and communication in autism. In F. R. Volkmar, R. Paul, A. Klin, \& D. Cohen (Eds.), Handbook of autism and pervasive developmental disorders, 335-364. Hoboken, NJ: John Wiley \& Sons. 
Tomasello, M. and Mervis, C.B., (1994). The instrument is great, but measuring comprehension is still a problem. Monographs of the Society for Research in child Development, 59(5), 174-179.

Volden, J. (2017). Autism spectrum disorder. In: Cummings, L. (ed). Research in clinical pragmatics. Cham: Springer International Publishing, 59-83.

Volden, J., Coolican, J., Garon, N., White, J., \& Bryson, S. (2009). Brief report: pragmatic language in autism spectrum disorder: relationships to measures of ability and disability. Journal of Autism and Developmental Disorders, 39(2), 388-393.

Volden, J., \& Phillips, L. (2010). Measuring pragmatic language in speakers with autism spectrum disorders: comparing the children's communication checklist-2 and the test of pragmatic language. American Journal of Speech - Language Pathology (Online), 19(3), 204-212A.

Volden, J. (2004). Conversational repair in speakers with autism spectrum disorder. International Journal of Language and Communication Disorders, 39(2), 171-189.

Volkmar, F., Carter, A., Grossman, J. \& Klin. (1997). Social development in autism. In: Cohen, D. \& Volkmar, F. (eds). Handbook of autism and pervasive developmental disorders. 2nd ed. Hoboken, NJ: John Wiley \& Sons.

Whitman, T. L. (2004). The development of autism: A self-regulatory perspective. Jessica Kingsley Publishers.

Whitmire, K.A. (2000). Adolescence as a developmental phase: a tutorial. Topics in Language Disorders, 20 (2), 1-14.

Young, E. C., Diehl, J. J., Morris, D., Hyman, S. L., \& Bennetto, L. (2005). The use of two language tests to identify pragmatic language problems in children with autism spectrum disorders. Language, Speech, and Hearing Services in Schools, 36(1), 6272.

Zufferey, S. (2015). Acquiring pragmatics: social and cognitive perspective. London and New York: Routledge. 